\title{
Medical Monitoring Model of Internet of Things Based on the Adaptive Threshold Difference Algorithm
}

\author{
Beibei Dong, Jingjing Yang, Yanli Ma and Xiao Zhang* \\ The College of Information Science and Engineering, Hebei North University, \\ Zhangjiakou Hebei, 075000, China. \\ *Corresponding author \\ E-mail:xz1965cn@aliyun.com
}

\begin{abstract}
There are still problems such as low detection accuracy and poor noise immunity in the application of the standard threshold difference algorithm in the signal detection of electrocardiosignal (ECG), in this paper, a medical monitoring model based on the adaptive threshold difference is proposed. First we use a nonlinear filter to filter the $P$ wave and $T$ wave which are low frequency in ECG signal. Then complex wave QRS will be tested. Then the algorithm will be more accuracy through the detection of the $R-R$ interval length and the adjustment of threshold. Finally, the ECG signal will be test with quadratic spline wavelet twice, and the error judgment will be known through adaptive threshold difference. In the simulation experiments, after judging error by wavelet transformation and making the standard threshold difference algorithm optimize adaptively, algorithm showed excellent detection accuracy with and without noise.
\end{abstract}

Keywords: Difference threshold; Adaptive optimization; Quadratic spline wavelet; ECG signal; Medical monitoring

\section{Introduction}

In the traditional medical mode, the quality and scale of medical service can't meet the needs of patients [1]. It is of great significance to establish a set of family oriented remote medical surveillance system based on mobile Internet. The development of family oriented remote medical monitoring system based on mobile Internet, and replacing people with more intelligent equipment greatly reduce the cost of medical [2]. Services of health care can also be provided to more people, can make more people get real-time monitoring and reduce costs of health care. Then the problem of "medical treatment is difficult and expensive" will slow down. It can also enhance people's health awareness, improve the quality of life and maintain social harmony and stability [3].

At present, the world's major developed countries are concerned about the construction of the Internet of things technology in the health field. FDA take a lot of practical actions to promote the implementation and promotion of RFID, and even making legislation to strengthen the application of RFID technology in drug transportation, sales, anticounterfeiting, tracking system [4]. Competent authorities of Japanese information communications industry proposed the development "U-Japan strategy" of IT industry. One of the purposes of the strategy is to promote the reform of the medical system, and to solve the medical and health problems of the society which is aged and lacking of kids [5] South Korea proposed the u-Korea strategy, which refers to the establishment of intelligent society through information technology in the future, so that people can enjoy the medical services anytime and anywhere [6]. The concept of "medical wisdom" was introduced by IBM. In order to realize the connection of medical information, sharing, collaboration, and public health prevention, they envisaged to introduce technology about Internet into the medical field, and think that IOT technology will be extensive used in the 
field of medical platform, electronic health records system and other fields [7]. The European Commission released the IOT strategy in form of the policy document, and wants to make Europe the global leader in the infrastructure which is based on Internet intelligent development. They also invest 4 billion euros through the ICT, and the money is used to launch more than 90 related researches and development projects. In addition to improve the network intelligent level, they invest new 2 billion euros annual in 2011 to 2013 to further strengthen the research and development efforts, also set aside 3 billion euros for construction of short-term projects which is related with IOT, including projects related medical. In recent years, the Chinese government also attaches great importance to the application of IOT technology in the medical field [8]. In 2008, in order to put forward the one-card process, expand the IC card in the medical field service scope and establish supervision and tracing system of RFID, the Chinese government published "development planning of the use of IC card in the health system during the 11th Five Year Plan", and want to strengthen the connection of medical industry and banks. Then we can put forward the process of the development of medical information system and the process of combination of IC card and RFID [9]. In April 6, 2009, the CPC Central Committee and the State Council promulgated "the further reforming opinions about the medical and health system". In order to ensure the success of medical reform, the government at all levels will invest 850000000000 yuan to strengthen the construction of medical information system, especially the application of RFID technology in medical field within 3 years. In May 23, 2009, the Ministry of health held a meeting about application of RFID technology. The meeting focused on the medical equipment management, drugs, blood and hygienic materials in the field of RFID applications [10]. In "The outline of development of health information", RFID technology and IC card were included in the overall program of the Ministry of health information construction. At present, the relevant departments of the country are speeding up the development format standards, capacity standards and information standards of IC card in medical field, and actively promote the application of IC cards and the Sharing of medical information [11].

According to problem in ECG signal detection of standard threshold difference algorithm, this paper presents medical monitoring model based on adaptive threshold optimization of network, and simulate the result to verify the validity of the model.

\section{ECG Signal Detection Model Based on Threshold Difference}

We know that we can get the limit value through the certain function (1) between the point $x+h$ and the point $x$ in first order differential:

$$
f^{\prime}(x)=\frac{d f(x)}{d x}=\lim _{h \rightarrow 0} \frac{f(x+h)-f(x)}{(x+h)-x}
$$

If the consecutive points were replaced by discrete points which is $x_{0} x_{1} x_{2}, \ldots, x_{k-1} x_{k} x_{k+1}, \ldots$. Then the calculation of discrete point is the first difference (2).

$$
f^{\prime}\left(x_{k}\right)=\frac{f\left(x_{k+1}\right)-f\left(x_{k}\right)}{x_{k+1}-x_{k}}
$$

There is $h=x_{k+1}-x_{k}$ above. The upper type is called forward differential, because the other point $x_{k+1}$ is in front of the reference point $x_{k}$. The geometric meaning of the formula is that we can use value of two points $f\left(x_{k+1}\right)$ and $f\left(x_{k}\right)$ to calculate the slope at the point $x_{k}$, and we can use this feature to determine the local extreme points of $f(x)$. In fact, $f\left(x_{k}\right)$ can also be calculated in the following two types (3) and (4):

Backward difference 
Central difference

$$
f^{\prime}\left(x_{k}\right)=\frac{f\left(x_{k}\right)-f\left(x_{k-1}\right)}{x_{k}-x_{k-1}}
$$

$$
f^{\prime}\left(x_{k}\right)=\frac{f\left(x_{k+1}\right)-f\left(x_{k-1}\right)}{x_{k+1}-x_{k-1}}
$$

Higher difference can be calculated by lower difference, such as the steps below:

$$
f^{\prime}(x)=\frac{d f^{\prime}(x)}{d x}
$$

The difference formulas are (5), (6)and (7).

Forward difference

$$
f^{\prime \prime}=\frac{f^{\prime}\left(x_{k+1}\right)-f^{\prime}\left(x_{k}\right)}{x_{k+1}-x_{k}}
$$

Backward difference

$$
f^{\prime \prime}=\frac{f^{\prime}\left(x_{k}\right)-f^{\prime}\left(x_{k-1}\right)}{x_{k}-x_{k-1}}
$$

Central difference

$$
f^{\prime \prime}=\frac{f^{\prime}\left(x_{k+1}\right)-f^{\prime}\left(x_{k-1}\right)}{x_{k+1}-x_{k-1}}
$$

The geometric meaning of the second order difference is to use difference of $f^{\prime}\left(x_{k+1}\right)$ and $f^{\prime}\left(x_{k}\right)$ to calculate the local maximum value and local minimum value at the inflection point $x_{k}$.

The method of difference threshold in ECG signal detection is to use differential operation to separate signal into first order and second order differential, and to establish the corresponding relationship between the singular point and the original signal. As a matter of fact, the ECG signal $R$ has large amplitude shock wave and greater slope. The location of the wave $R$ can be ensured through the change of slope. Then set appropriate threshold value based on the test to ensure the exact location of wave $R$. Then other waveform will be easy to test.

Difference method is a simple and fast algorithm, suitable for requirements of higher ECG monitor in real time, but its ability of anti-interference is poor and motion artifacts in signal detection efficiency will decrease. So it is better to filter the wave and then do the difference method.

\section{Medical Monitoring Model Based on Adaptive Threshold Difference}

\subsection{Detection of QRS Complex Wave Based on Adaptive Threshold Difference}

Some results can be realized through the analysis of the square of first and second difference. After a series of dispose, original complex wave of electrical signal QRS is higher than other waves which make it different with other characteristic waves in the series of waves $F(n)$. That means let the signal go through a nonlinear filter, and the low frequency waves such as $\mathrm{P}$ wave and $\mathrm{T}$ wave will be filtered. Based on the detection of QRS, the interference from $\mathrm{P}$ wave and $\mathrm{T}$ wave or other non ECG QRS complex waves can be effectively reduced.

Then, the threshold value will be set according to the new data sequence of $F(n)$. The location of $\mathrm{R}$ wave will be ensure according to whether the $F(n)$ is larger than threshold 
value. The initial value of $R_{t h}$ is $A_{0}$, and there is $A_{0}=\frac{1}{4} \max \{F(n)\}$. The existence of $\mathrm{R}$ wave can be known by follows: If there are more than three times which $F(i)>A_{0}$ appears when there is $i \in I$. Then the wave QRS will be found in electrical signal $f(n)$ in section $I$. The maximum value in a certain section in the middle of $I$ is regarded as the wave crest of $\mathrm{R}$. In each sequence of data, the first point is the starting point of the $\mathrm{R}$ wave, and the final point of the $\mathrm{R}$ wave is the end point, when there is $F(i)>A_{0}$.

$\mathrm{Q}$ wave and $\mathrm{S}$ wave is first trough before and after $\mathrm{R}$ wave. The $\mathrm{Q}$ valley point is minimum point value which is $20 \%$ of $T_{R R i}\left(T_{R R i}\right.$ is the former $\mathrm{RR}$ interval of the $\mathrm{R}$ wave) within the range of the wave valley, and the $\mathrm{S}$ valley point is minimum point value which is $35 \%$ of $T_{R R i+1}\left(T_{R R i+1}\right.$ is the next RR interval of the $\mathrm{R}$ wave) within the range of the wave valley. The position of $\mathrm{Q}$ wave and $\mathrm{S}$ wave is shown in Figure 1. Then the starting point and the ending point of $\mathrm{Q}$ wave and $\mathrm{S}$ wave through the slope of threshold value.

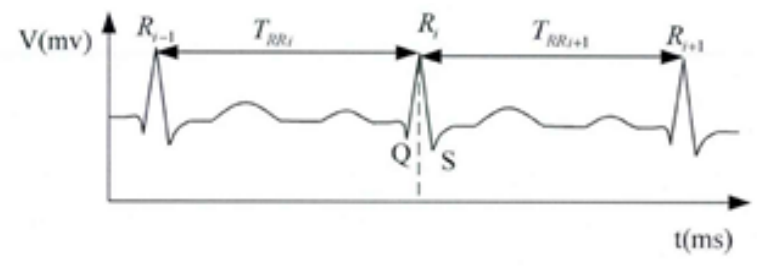

Figure 1. Location Q-Wave and S-Wave

The algorithm has a high detection rate in the environment of high SNR, but there are also obvious shortcomings. The accuracy of the detection of QRS complex wave is largely determined by the effect of the signal preprocessing. If in pre-processing stage do not inhibit the noise, noise can be amplified in accordance with the above algorithm for signal amplification. Under strong electrical interference, it is easy to cause the error detection. When the complex QRS is relatively flat, some waves may be missed.

According to the situation above, the variable threshold is brought in the arithmetic, which is based on the length of interval $R-R$. The idea of this algorithm is to set an initial value $A_{0}$ for the threshold $R_{t h}$ and there is $A_{0}=\frac{1}{4} \max \{F(n)\}$. If there are more than three times which $F(i)>A_{0}$ appears when there is $i \in I$. Then the wave QRS will be found in electrical signal in section $I$. The maximum value in a certain section which is in the middle of $I$ is regarded as the wave crest $R_{i}$ of wave $\mathrm{R}$ and regarded as one value of sequence of $R(k)$ which will be test. Then other values in disorder sequence $F(n)$ will be test.

Then the time length of $T_{R R}$ ( $T_{R R}$ is RR interval), will be known through the calculation of two peaks of $R_{i}$ wave and $R_{i+1}$ wave. If there is $T_{R R}\left(T_{R R}\right.$ is RR interval) $>1.5 \bar{T}_{R R}\left(\bar{T}_{R R}\right.$ is RR interval average value of sequence of $R(k)$ ), that some conditions may be missing. Now there is threshold $R_{t h}=\frac{1}{2} A_{0}$. Then repeat the test process of $F(n)$ and sequence $R^{\prime}(k)$ obtained at this time is considered to be the final $\mathrm{R}$ wave position sequence. That means there exists bradycardic events in section data at this time. If there is $T_{R R}<\bar{T}_{R R} / 1.5$. That means error detection may exist. Then there is threshold $R_{t h}=2 A_{0}$. The test process of $F(n)$ will be repeated and sequence $R^{\prime}(k)$ which we get in the test will be considered as the final $\mathrm{R}$ wave position sequence. That means there exists bradycardic events in section data at this time. The $R(k)$ is regarded as the final wave $\mathrm{R}$ if there are no above two cases. 


\subsection{Error Determination Based on Wavelet Transformation}

Spline wavelet have the feature of minimal support set, orthorhombic symmetry, good frequency characteristic, good resolution, bands of small coherent, smooth and so on, thus it becomes optimal signal wavelet mutation detection. In this paper, the second spline wavelet $\psi(t)$ is selected to test wave $\mathrm{R}$, and its frequency domain expression is:

$$
\hat{\psi}(\omega)=i \omega\left[\frac{\sin \left(\frac{\omega}{4}\right)}{\frac{\omega}{4}}\right]^{4}
$$

The function of the second spline wavelet:

$$
\left\{\begin{array}{l}
H(\omega)=e^{\frac{i \omega}{2}}\left[\cos \left(\frac{\omega}{2}\right)\right]^{3} \\
G(\omega)=4 i e^{\frac{i \omega}{2}} \sin \left(\frac{\omega}{2}\right)
\end{array}\right.
$$

The characteristic scale: The small scale is the high frequency component of the signal, and the large scale is corresponding to the low frequency component of the signal. After ECG signal is separated by wavelet, the energy distribution is different due to frequency of the characteristic waves. The energy of QRS wave is focused on $2^{3}$ and $2^{4} .2^{3}$ has the maximum energy. The energy of QRS wave group are gradually reduced when the center is $2^{3}$. The high frequency $\mathrm{Q}$ and $\mathrm{S}$ waves in the ECG signal are the most obvious modulus maxima pair at $2^{1}$ and $2^{2}$. And the low frequency $\mathrm{P}$ wave and $\mathrm{T}$ wave are the most obvious modulus maxima pair $2^{4}$ and $2^{5}$. For the larger scales $j(j>5)$, The feature of positive and negative extrema of ECG signal is not obvious, but other interfering is heavy, and calculation is hard. So this paper shows how to extract ECG characteristic waveform based on former 5 scales.

How to determine $\mathrm{R}$ wave mode maxima: $\mathrm{R}$ wave has a pair of modulus maxima on the each characteristics scale. In the situation of frequency noise, its maxima distribute at small scales is small or non-existent in large scale. For low frequency signals such as $\mathrm{P}$ wave and $\mathrm{T}$ wave is on the contrary, their maxima are mainly distributes in large scale, on a small scale is small or does not exist. This shows that high frequency noise or $\mathrm{P}$ wave and $\mathrm{T}$ wave cannot produce maxima. So it is easy to reduce the affection of noise and other waves through the test of characteristic wave of $\mathrm{R}$.

The maximum of the modulus generated at the scale $2^{3}$ of $\mathrm{R}$ wave is the maximum. The zero value of the middle of positive maximum and negative maximum is the location of the R peak. For the binary wavelet transform, the signal singular points correspond to the wavelet transform $2^{1}$ scale positive-negative value of the zero crossing point, and there is a time offset which is $\left(2^{j}-1\right) / 2$ at the zero point of signal singularity.

The main processes of the detection of $\mathrm{R}$ wave in $2^{3}$ scale are as follows:

(1) $W 2^{j} f(n)$ will be get through the decomposition of the ECG signal $f(n)$ with twospline wavelet;

(2) Ensure the positive and negative thresholds $A_{1}$ and $A_{2}$ in $W 2^{3} f(n)$;

(3) According to $A_{1}$ and $A_{2}$ in the upper (2) to get the positive maximum and negative maximum and ensure positive maximum $>A_{1}$ and negative maximum $<A_{2}$.

(4) Ensure the zero crossing point between the positive electrode value and the negative value pair, that is the crest position of $R$ wave;

(5) Delete redundant $\mathrm{R}$ peak and compensate the value of $\mathrm{R}$ peak; 
(6) Modify the test with time shifting $\left(2^{j}-1\right) / 2$ at the crossing point of signal singular point and the corresponding zero point.

In order to improve the detection rate of $\mathrm{R}$ wave, an adaptive method is proposed to determine the detection threshold $A_{1}$ and $A_{2}$ of $\mathrm{R}$ wave. Specific process: The first 14 seconds of ECG data is divided into seven sections which has 2 seconds for a section. Then positive and negative mold maximum will be known. Then remove maximum and minimum value in direction to respectively. Then get the average of the left value. Positive and negative mold maximum $M$ and $N$ will be known. The initial threshold $A_{1}$ and $A_{2}$ were regarded as their $3 / 4$ average value of the positive maximum-the negative value. We can use the result above and the former pair of threshold. The position of the adaptive threshold can detect location of $\mathrm{R}$ peak more precisely.

The right $\mathrm{R}$ wave is detected when the results of adaptive difference threshold method and adaptive wavelet threshold algorithm are the same. When the results are different, another test should be done before and after 4 seconds each time window at the certain point. Combined with the above two methods, the position of $\mathrm{R}$ peak can be detected more accurately.

\section{Algorithm Performance Simulation}

In order to validate the feasibility of the proposed algorithm performance, and carry on the simulation experiment, we take two medical monitoring of the ECG signal as the example, and use the method to test. The ECG signal of first medical care without any noise shows like the following chart.

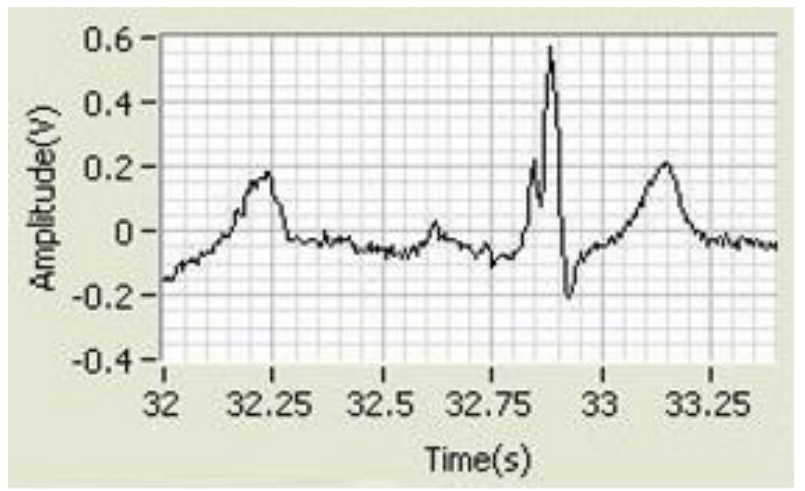

Figure 2. Standard Algorithm to Track the Results of Unobstructed Case

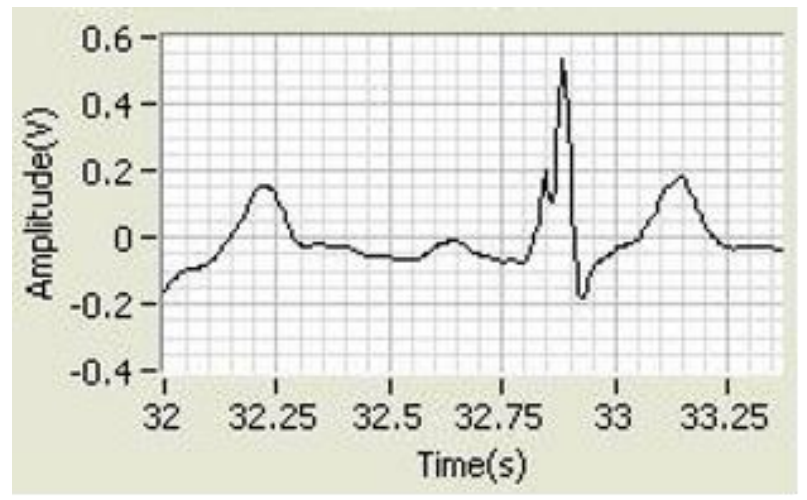

Figure 3. Improved Algorithm Test Results Noiseless Case

In the second medical care, the ECG signal is added to Gaussian noise, the comparation of standard threshold difference algorithm and the detection and the 
adaptive optimized threshold difference algorithm proposed in this paper like the following chart.

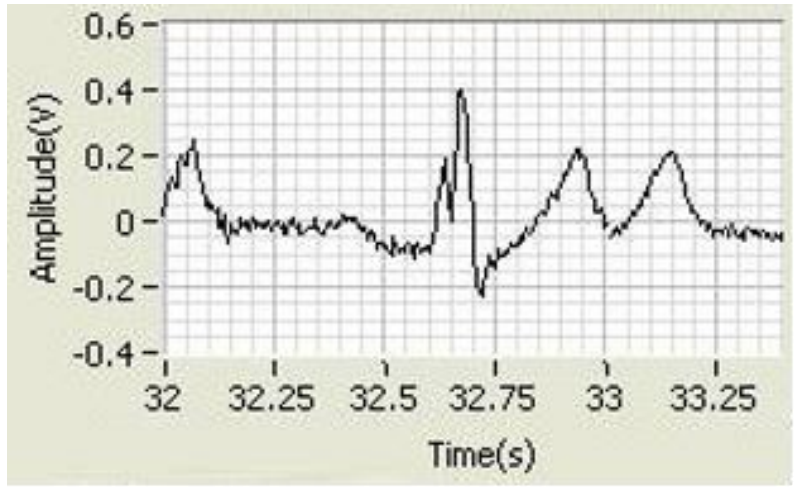

Figure 4. Standard Algorithm Test Results Noise Case

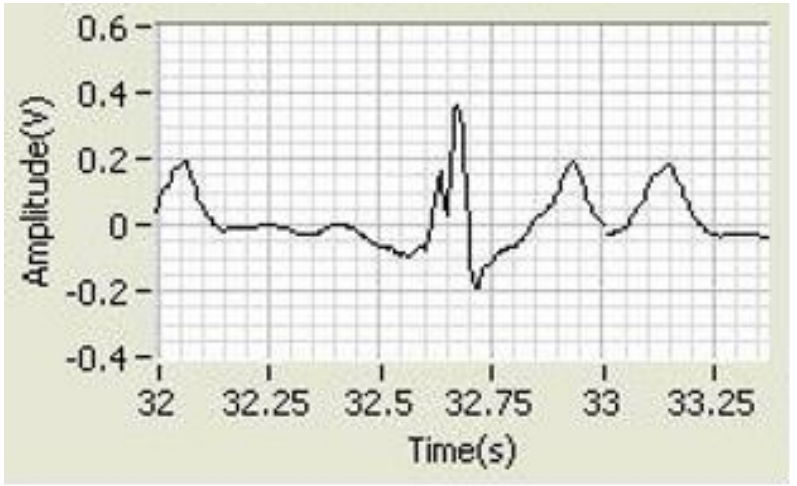

Figure 5. Results Improved Algorithm Detect Noise Case

From the results, we can know through standard threshold difference algorithm of adaptive optimization, and using wavelet transform to determine error with and without noise, we can improve accuracy of the result.

\section{Summary}

In recent years, with the rapid development of computer communication technology, wireless sensor network technology, as well as the emergence of new concepts such as Internet of things and cloud computing, the research on the key technology of telemedicine becomes the hot research direction. The introduction of these new technologies will help telemedicine service experience to upgrade through the biomedical sensor into a more miniaturized, intelligent direction. Remote medical care for the sudden and chronic diseases of the guardianship has a very important significance, so that realtime monitoring has become popular and simple. According to standard threshold, the detection accuracy of the test is improved with and without noise.

\section{Acknowledgment}

This work was supported by:

1. Youth Foundation of the Education Department of Hebei Province (QN2014182)

2. Major project of Hebei North University, Hebei Province Population Health Information Engineering Technology Research Center (ZD201301)

3. Youth Foundation of the Education Department of Hebei Province (QN2015225)

4. Science and Technology Department of Hebei Province (112135119) 
5. Youth Foundation of Natural Science of Hebei North University (Q2014008)

6. Zhangjiakou Department of Science and Technology Project(1421012B)

7. Research and Design for Prairie Wireless Fire Alarm System Based on ZigBee (1411073B)

\section{References}

[1] C. Zhangjin, "Design of Medical Infusion Monitoring System Based on ZigBee Technology", Computer Measurement \& Control, vol. 23, no. 3, (2015), pp. 797-800.

[2] C. Hongling, "Realization of the Remote Medical Monitoring and Diagnostic Heterogeneous System Integration", Computer Measurement \& Control, vol. 22, no. 12, (2015), pp. 3929-3931.

[3] Z. Lin, "Research and Design of Wireless Medical Monitoring System Based on ZigBee", Tv Engineering, vol. 37, no. 14, (2015), pp. 64-67.

[4] L. Shanghui, "Development and Application of Family Telemedicine Monitoring System", Chinese General Practice, vol. 15, no. 13, (2015), pp. 1542-1542.

[5] H. Fengjun, Z. Yanwei and C. Jian, "SIFT Feature Points Detection and Extraction of ThreeDimensional Point Cloud", WIT Transactions on Information and Communication Technologies, no. 60, (2014), pp. 603-611.

[6] H. Fengjun and Z. Yanwei, "Comparative research of matching algorithms for stereo vision", Journal of Computational Information Systems, vol. 9, no. 13, (2013), pp. 5457-5465.

[7] Z. Jinmeng, "Research of ZigBee- based Medical Monitoring Network Oriented to Family", Computer Measurement \& Control, vol. 20, no. 3, (2015), pp. 780-783.

[8] T. Qiaolin, "Design and implementation of the remote health care system based on GPS_GPRS_GIS", Science of Surveying and Mapping, vol. 36, no. 6, (2014), pp. 216-218.

[9] Z. Xiang, "Design of an Embedded ZigBee Remote Medical Monitoring System", Journal of Southwest Agricultural University, vol. 33, no. 3, (2011), pp. 158-162.

[10] L. Hongqiang, "Research on key technologies of ECG monitoring system based on Internet of things", Application Research of Computers, vol. 51, no. 12, (2014), pp. 4600-4603.

[11] X. Dahua, "Intelligent Medical WardShip-System based on Agent", Laboratory Research and Exploration, vol. 27, no. 11, (2014), pp. 41-44. 